\title{
An Interval Type-2 Fuzzy $K$-Nearest Neighbor
}

\author{
Frank Chung-Hoon Rhee and Cheul Hwang \\ Computation Vision and Fuzzy Systems Laboratory \\ Department of Electronic Engineering \\ Hanyang University \\ Ansan, Korea \\ \{frhee,chwang\}@fuzzy.hanyang.ac.kr
}

\begin{abstract}
This paper presents an interval type-2 fuzzy $K$ nearest neighbor (NN) algorithm that is an extension of the type1 fuzzy $K$-NN algorithm proposed in [1]. In our proposed method, the membership values for each pattern vector are extended as interval type-2 fuzzy memberships by assigning uncertainty to the type-1 memberships. By doing so, the classification result obtained by the interval type-2 fuzzy $K$-NN is found to be more reasonable than that of the crisp and type-1 fuzzy $K$-NN. Experimental results are given to show the effectiveness of our method.
\end{abstract}

\section{INTRODUCTION}

The conventional (crisp) $K$-NN is a simple algorithm that is used to assign patterns of unknown classification to the class of the majority of its $K$ nearest neighbors of known classification according to a distance measure [3]. However, a major drawback of the method is that each of the patterns of known classification is considered equally important in the assignment of the pattern to be classified. This can cause difficulties in regions where pattern data overlap. To overcome this drawback, a fuzzy version of the $K$-NN was proposed [1]. In this method, the assigned memberships play a role in the amount of contribution of a pattern during the classification process. As a result, the selected pattern that has low membership suggests small contribution on the classification for the pattern [1]. Therefore, this can allow the misclassification rate to decrease even for class pattern data that overlap. However, the result may still be sensitive to the selection of $K$. Poor selection of $K$ can result in an undesirable classification rate. Therefore, we focus on the uncertainty involved in the selection of $K$. This uncertainty is represented by an interval between the memberships that consider several values of $K$ for the initial fuzzification process. As a result, we propose an interval type-2 fuzzy $K$ NN.

The extension to the interval type-2 fuzzy case revises the initial fuzzification process in the fuzzy $K$-NN algorithm. In the type-1 fuzzy case, only one initial $K$ is selected to assign initial fuzzy memberships to the pattern data. If the selection of $K$ is poor, an undesirable classification rate for the pattern data can be obtained. However, for the interval type-2 fuzzy approach, we need not select only one initial $K$. This is due to the extension of the pattern data into an interval type-2 fuzzy set.

This work was supported by Hanyang University, Korca, made in the program year of 2002 .
For this extension, we use initial $K$ values in an appropriate range. Handling of this uncertainty can decrease the contribution of an undesirable initial $K$ on the classification process for the patterns. Hence, this can provide a more reasonable classification result by managing the uncertainty for the selected initial $K$.

In summary, our proposed interval type-2 fuzzy approach possesses the following advantages.

1) Due to the interval type-2 fuzzy extension, we need not choose only one initial $K$.

2) By considering various initial $K$ values for the pattern memberships, a more reasonable classification result may be obtained.

The remainder of this paper is organized as follows. In Section 2, we briefly describe the crisp and fuzzy $K$-NN algorithms. In Section 3, we describe how to extend type-1 fuzzy memberships to interval type-2 fuzzy memberships for use in our proposed method. In Section 4, we describe our interval type-2 fuzzy $K$-NN. Section 5 gives some experimental results that are applied to our method. Finally, Section 6 gives the conclusions.

\section{CRISP AND FuZZY $K$-NN}

Nearest neighbor classifiers require no preprocessing of the labeled pattem set prior to their use. The crisp 1-nearest neighbor classification rule assigns a given input pattern, which is of unknown classification, to the class of its nearest neighbor [1], [3]. This idea can be extended to $K$-nearest neighbors with the given pattern being assigned to the class that is represented by a majority among the $K$-nearest neighbors. This can be summarized as follows.

\section{A. The crisp K-NN algorithm}

Let $W=\left\{x_{1}, \ldots, x_{n}\right\}$ be a set of $n$ labeled patterns;

\section{BEGIN}

Input $y$, of unknown classification

Set $K, 1 \leq K \leq n$

Initialize $i=1$

DO UNTIL ( $K$-nearest neighbors are found)

Compute distance from $y$ to $x_{i}$ :

IF $(i \leq K)$ THEN

Include $x_{i}$ in the set of $K$-nearest neighbors

ELSE IF $\left(x_{i}\right.$ is closer to $y$ than any previous nearest neighbor) THEN
The IEEE International Conference on Fuzzy Systems 

neighbors;

Delete farthest pattern in the set of $K$-nearest Include $x_{i}$ in the set of $K$-nearest neighbors;

\section{END IF}

Increment $i$

END DO UNTIL

Determine the majority class represented $i$ the set of $K$ nearest neighbors

IF (a tie exists) THEN

Compute sum of distances of neighbors in each class that tied

IF (no tie occurs) THEN

Classify $y$ in the class of minimum sum

ELSE

Classify $y$ in the class of last minimum found

END IF

ELSE

Classify $\boldsymbol{y}$ in the majority class

END IF

\section{END}

\section{B. The fuzzy $K-N N$ algorithm}

The fuzzy $K$-nearest neighbor algorithm assigns class membership to a pattern rather than assigning the pattern to a particular class. The membership values for the pattern should provide a level of assurance to accompany the resultant classification. The basis of the algorithm is to assign membership as a function of the pattern distance from its $K$ nearest neighbors and those neighbors' memberships in the possible classes [1]. The assigned membership of the pattern $\boldsymbol{x}$ is computed as

$$
u_{i}(x)=\frac{\sum_{j=1}^{K} u_{i j}\left(1 /\left\|x-x_{j}\right\|^{2 /(m-1)}\right)}{\sum_{j=1}^{K}\left(1 /\left\|x-x_{j}\right\|^{2 /(m-1)}\right)},
$$

where $u_{i j}$ is the membership in the $i$ th class of the $j$ th pattern of the labeled pattern set.

As seen in (1), the assigned memberships of pattern $x$ are influenced by the inverse of the distances from the nearest neighbors and their class memberships. The inverse distance provides more weight to a pattern's membership if it is closer and less if it is farther from the pattern under consideration. In addition, the labeled patterns can be assigned class memberships in several ways. As in [1], a reasonable membership assignment in each class can be computed as

$$
u_{j}=\left\{\begin{array}{ll}
0.51+\left(n_{j} / K\right)^{*} 0.49, & \text { if } j=i \\
\left(n_{j} / K\right)^{*} 0.49, & \text { if } j \neq i
\end{array},\right.
$$

where $n_{j}$ denotes the number of neighbors which belong to the $j$ th class.

The fuzzy algorithm is similar to the crisp version in the sense that it must also search the labeled pattern set for the $K$ nearest neighbors. Beyond obtaining these $K$ patterns, the procedures differ considerably. The algorithm can be summarized as follows.

Let $W=\left\{x_{1}, \ldots, x_{n}\right\}$ be a set of $n$ labeled patterns. The algorithm is as follows [1]:

\section{BEGIN}

Input $x$, of unknown classification

Set $K, 1 \leq K \leq n$

Initialize $i=1$

DO UNTIL ( $K$-nearest neighbors to $x$ found)

Compute distance from $\boldsymbol{x}$ to $\boldsymbol{x}_{\boldsymbol{i}}$

IF $(i \leq K)$ THEN

Include $x_{i}$ in the set of $K$-nearest neighbors

\section{END IF}

\section{END DO UNTIL}

Initialize $i=1$

DO UNTIL ( $x$ assigned membership in all classes)

Compute $u_{i}(x)$ using (1)

Increment $i$

\section{END DO UNTIL}

END

\section{EXTENSION TO INTERVAL TyPE-2 FuZZy SET}

In Fuzzy $K$-NN, we extend the pattern set to fuzzy set before attempting this method. For this, it is necessary to select an appropriate initial $K$ for the initialization process. Unfortunately, it is difficult to determine which $K$ is most desirable for the given pattern set. This suggests that uncertainty is present in the selected initial $K$. In [1], various results can be obtained by varying $K$. As shown by various results, selection of an undesirable initial $K$ can cause an undesirable misclassification rate. In this paper, several initial $K$ values are used to manage and control the uncertainty [2], [4]-[6]. From this point of view, we extend the pattern data to interval type- 2 fuzzy sets. At first, we assign several primary membership values to a given pattern set. For this, we consider several number of initial $K$ value for the initialization process, namely, fuzzy $K$-INIT [1]. This is used for determining the elements of primary memberships on interval type-2 fuzzy set.

In this process, we cannot obtain $N$ primary membership values although we use $N$ numbers of initial $K$. This is due to regions of non-overlapping patterns. If we select initial $K$ value in an appropriate range, a given pattern guaranteed to have full membership for any initial $K$ value is selected. The following example illustrates this.

In Fig. 1, suppose that pattern "square" belongs to class 1 and "triangle" belongs to class 2 . For the pattern that is indicated as " $x_{1}$," it always has full membership for class 1 , and zero membership for class 2 when initial $K$ is 1 or 2 . In this case, the primary membership sets for pattern " $x_{1}$ ", becomes

$$
u_{1}\left(x_{1}\right)=\{1.0\}, u_{2}\left(x_{1}\right)=\{0.0\}
$$

Although we use 2 values for initial $K$, we can respectively obtain a primary membership element for each class. 


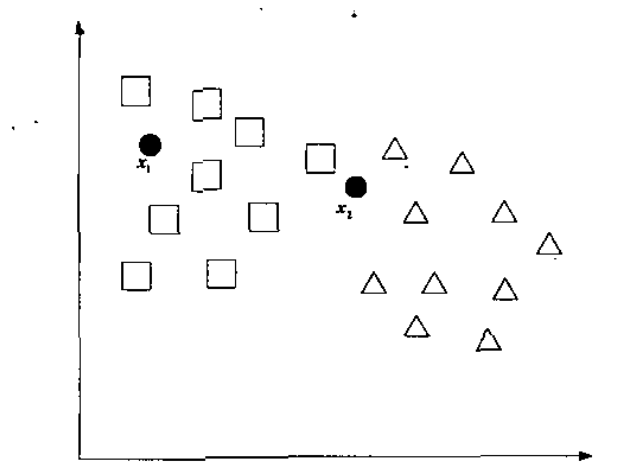

Fig. 1. An example of membership assignment by selecting initial $K$ value and location of pattern

However, for another pattern that is indicated as " $x_{2}$," a different result is obtained when initial $K$ is chosen as 2 . As seen in Fig. 1, the pattern has full membership for class 1 when initial $K$ is 1 . However, by increasing the initial $K$, the membership of the pattern for class 1 decreases and the membership of the pattern for class 2 increases. Therefore, when we use several initial $K$ values to extend to interval type-2 fuzzy sets, we can be obtained several primary membership values for each class. For example, we can obtain the following result for the pattern " $x_{2}$," if we use initial $K=1,2,3$.

$$
u_{1}\left(x_{2}\right)=\{1.0,0.755,0.674\}, u_{2}\left(x_{2}\right)=\{0,0.245,0.326\}
$$

From this result, we can derive a specific relation between initial $K$ and primary membership as

$$
\begin{aligned}
& 1 \leq \text { element number of } \bigcup_{r=1}^{N} u\left(j, K_{r}, x_{j}\right) \leq N \\
& \text { where } K_{r}=\left\{K_{1}, \cdots, K_{N}\right\}
\end{aligned}
$$

In (5), $u\left(j, K_{r}, x_{i}\right)$ represents the primary membership of the $i$ th pattern in class $j$ when initial $K$ is selected as $K_{r}$.

Extension to interval type-2 fuzzy sets by this method has the following property.

A pattern in a non-fuzzy framework remains as a type-1 fuzzy set, and a pattern in fuzzy framework extends to an interval type-2 fuzzy set.

Fig. 2 represents the extension to an interval type- 2 fuzzy set for each pattern in a fuzzy framework. As seen by Fig. 2 , we should use various initial $K$ values in an appropriate range for a given $x_{k}$. Next, we perform union operation by the membership values for a given $x_{k}$. In this process, we can reduce redundant primary membership values. After that, we can obtain primary memberships for a given $\boldsymbol{x}_{\boldsymbol{k}}$. Finally, we should consider secondary grade of each primary membership value. However, since we use an interval type-2 fuzzy set, we need not consider secondary grade. That is always 1.0 . Through this method, we can extend fuzzy set to an interval type-2 fuzzy set for $\boldsymbol{x}_{\boldsymbol{k}}$.

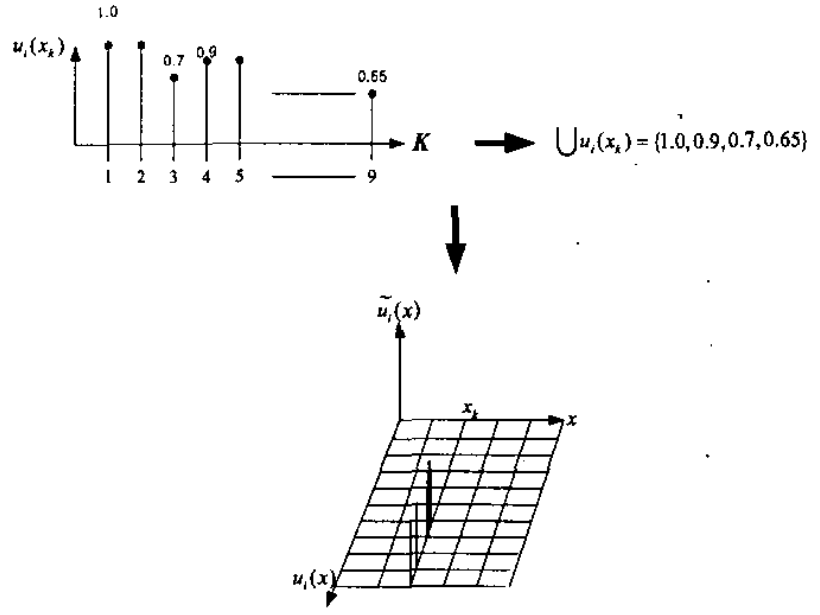

Fig. 2. An example of extension to interval type-2 fuzzy set for a pattern in the fuzzy domain

\section{INTERVAL TYPE-2 FUZZY $K$-NN}

Basically, we perform $K-\mathrm{NN}$ after extension to interval type-2 fuzzy set on initialization process. Therefore, when assigning interval type-2 fuzzy memberships for a given pattern, the membership assignment for each class [2], [4], [6] can be modified as

$$
\tilde{u}_{i}(x)=\frac{\sum_{j=1}^{K} \tilde{u}_{i j}\left(1 /\left\|x-x_{j}\right\|^{2 /(m-1)}\right)}{\sum_{j=1}^{K}\left(1 /\left\|x-x_{j}\right\|^{2 /(m-1)}\right)},
$$

where (6), $\tilde{u}_{i}$ represents the interval type-2 fuzzy membership of $\boldsymbol{x}$ for class $i$. In addition, $\tilde{u}_{i j}$ represent initial interval type- 2 fuzzy membership of $x_{j}$, which is the $j$ th nearest neighbor to $\boldsymbol{x}$ in class $i$. For calculating (6), we can consider embedded interval type- 2 fuzzy sets. This is explained as follows.

Suppose we consider $K=2$ then for two patterns that are nearest to a given $\boldsymbol{x}$, we can obtain embedded interval type- 2 fuzzy sets between the primary memberships of the 2 patterns. This follows the literature explained in [2], [4].

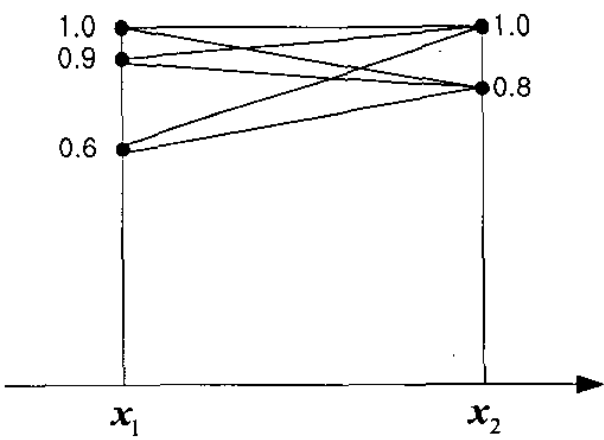

Fig. 3. An example of embedded interval type-2 fuzzy sets for the 2 pattems that are nearest to $x$. (i.e., $K=2$ ) 
Now, when we assume that each pattern hàs $n_{i}$ number of primary memberships, we can derive an equation which gives the number of primary membership for $x_{k}$ with the number of primary memberships for $K$ nearest neighbor patterns as

$$
\prod_{j=!}^{K} n_{j} \simeq R_{k i}
$$

where $k$ denotes the pattern number and $i$ denotes class number

From (7), we can represent the primary memberships of each pattern as

$$
u_{i}\left(x_{k}\right)=u_{i_{i k}}+\cdots+u_{R_{\text {d }}}=\sum_{r=1}^{R_{i k}} u_{r_{i k}}
$$

Now, we can extend to an interval type-2 fuzzy set for given pattern $x_{k}$ with the secondary membership of each primary membership as

$$
\begin{aligned}
& \tilde{u}_{i}\left(x_{k}\right)=f\left(u_{l_{i k}}\right) / u_{1_{i k}}+\cdots+f\left(u_{R_{i k}}\right) / u_{R_{i k}} \\
& =1.0 / u_{i_{i k}}+\cdots+1.0 / u_{R_{k}}=\sum_{r=1}^{R_{i k}} 1.0 / u_{r_{i k}}
\end{aligned}
$$

From (8) and (9), the given pattern remains as an interval type-2 fuzzy set. For the classification process by assigning memberships for a given pattern, the type-reduction process needs to be applied before defuzzification. For the typereduction process, the type-reduced membership of $x_{k}$ in class $i$ can be expressed as

$$
\bar{u}_{i}\left(x_{k}\right)=\frac{\sum_{r=1}^{R_{i k}} f\left(u_{r_{i k}}\right) u_{r_{i k}}}{\sum_{r=1}^{R_{i k}} f\left(u_{r_{i k}}\right)},
$$

where $\bar{u}_{i}\left(x_{k}\right)$ is type-reduced membership of $x_{k}$ in class $i$.

In (10), we need not consider the secondary grade since the secondary grade is always 1.0. Therefore, it is approximately the average of primary memberships of interval type-2 fuzzy set. In here, the membership of $\boldsymbol{x}_{\boldsymbol{k}}$ is no longer an interval type- 2 fuzzy set, we can estimate the degree of the pattern membership. we can classify a given pattern $x_{k}$ by membership values.

In the interval type- 2 fuzzy $K-\mathrm{NN}$, we can recognize that this method is equal to fuzzy $K$-NN method when a single initial $K$ is used. Also, when we set initial $K$ and $K$ equal to 1 , interval type-2 fuzzy $K-\mathrm{NN}$ is equivalent to the crisp $\mathrm{NN}$ method. Therefore, we can consider the interval type-2 fuzzy $K-\mathrm{NN}$ as a generalizing form of $K-\mathrm{NN}$.

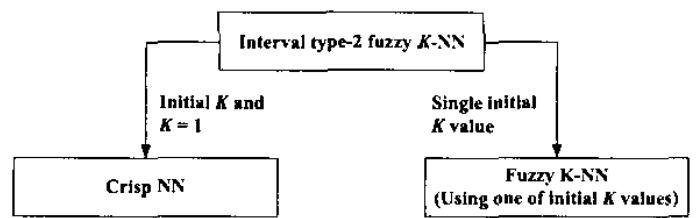

Fig. 4. Relationship between the crisp, fuzzy, and interval type-2 fuzzy approaches

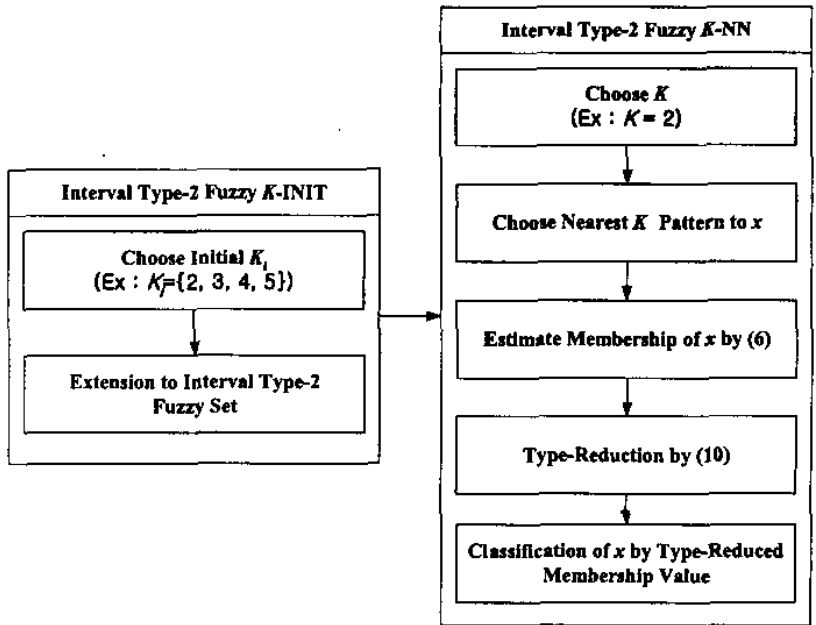

Fig. 5. Block diagram of the interval type-2 fuzzy $K-\mathrm{NN}$

Fig. 5 summarizes our interval type-2 fuzzy $K-\mathrm{NN}$ algorithm. As seen in Fig. 5, the algorithm consists of 2 stages. In the first stage, we perform interval type- 2 fuzzy $K$ INIT to extend pattern set to interval type-2 fuzzy sets. In the next stage, we perform interval type-2 fuzzy $K$-NN with interval type- 2 fuzzy set. In this part, we assign interval type2 fuzzy membership for a given pattern using the embedded interval type-2 fuzzy operation. Finally, when we classify pattern by membership grade, we perform type-reduction and defuzzification.

\section{EXPERIMENTAL RESULTS}

In this section, we first show an example using "twoclass" data by comparing results of the fuzzy and interval type-2 fuzzy $K$-NN methods. In doing so, we choose initial $K$ values $\{1,3,5,7,9\}$ and assign initial memberships for the pattern set. Also, we choose $K=\{1,2,3,4,5,6,7,8,9\}$ for each $K$ NN method. In other words, we perform the fuzzy $K-\mathrm{NN}$ method 5 times for each $K$, and interval type-2 fuzzy $K$-NN method 26 times for each $K$. As shown table I, we have 26 combination of the initial $K$ for interval type- 2 fuzzy $K-\mathrm{NN}$.

TABLE I

COMBINATION OF INTERVAL TYPE-2 FUZZY $K$-NN

\begin{tabular}{ccccc}
\hline $\begin{array}{l}\text { Number of } \\
\text { initial } K\end{array}$ & 2 & 3 & 4 & 5 \\
\hline & $\{1,3\}$ & $\{1,3,5\}$ & $\{1,3,5,7\}$ & $\{1,3,5,7,9\}$ \\
& $\{1,5\}$ & $\{1,3,7\}$ & $\{1,3,5,9\}$ & \\
& $\{1,7\}$ & $\{1,3,9\}$ & $\{1,3,7,9\}$ & \\
Elcment & $\{1,9\}$ & $\{1,5,7\}$ & $\{1,5,7,9\}$ & \\
Combination & $\{3,5\}$ & $\{1,5,9\}$ & $\{3,5,7,9\}$ & \\
& $\{3,9\}$ & $\{1,7,9\}$ & & \\
& $\{5,7\}$ & $\{3,5,9\}$ & & \\
& $\{5,9\}$ & $\{3,7,9\}$ & & \\
& $\{7,9\}$ & $\{5,7,9\}$ & & \\
\hline
\end{tabular}


Fig. 6 show the average number of misclassifications for "two class" data for the fuzzy and interval type-2 fuzzy $K-\mathrm{NN}$. As shown in Fig. 6, the interval type-2 fuzzy $K$-NN shows slightly better performance than the fuzzy $K-\mathrm{NN}$. This classification result for the interval type- 2 fuzzy $K-\mathrm{NN}$ can be obtained by the relatively low influence of the undesirable initial $K$. For this, we use several initial $K$ values for the interval type-2 fuzzy $K-\mathrm{NN}$.

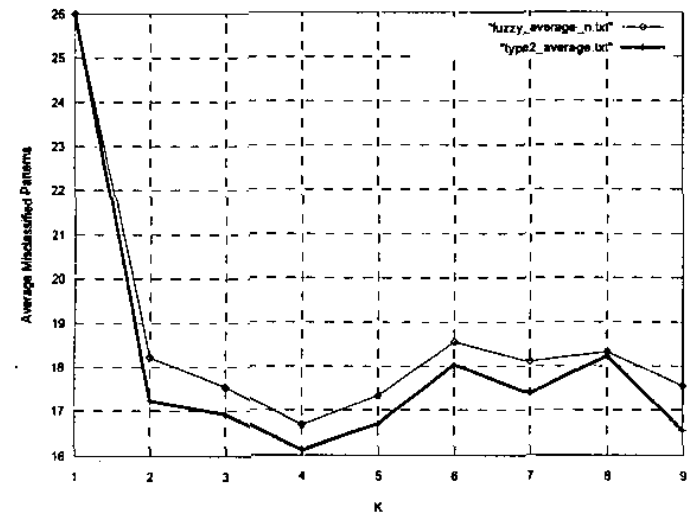

Fig. 6. Average number of misclassification for "twoclass" data

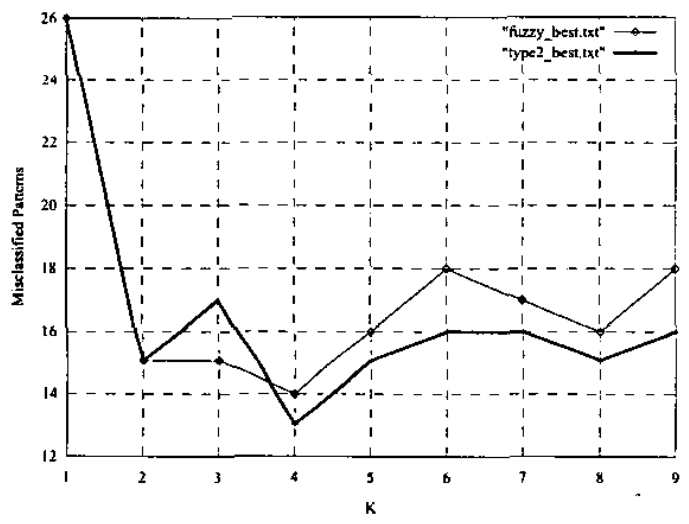

Fig. 7. Minimum misclassification rate using a fixed value for initial $K$

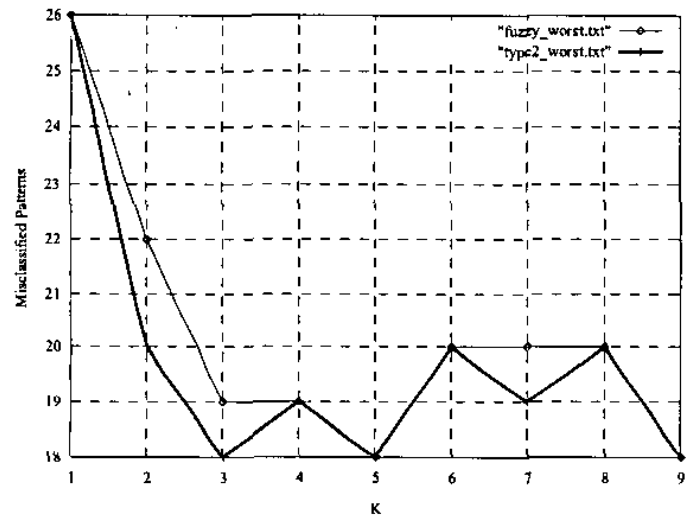

Fig. 8. Maximum misclassification rate using a fixed value for initial $K$
Fig. $7(8)$ shows the minimum (maximum) misclassification rate when we fix initial $K$ to a specified value.

We now show a more reliable classification result in our next example. Fig. 9 shows the scatter plot of the "T-shape" data used for our example.

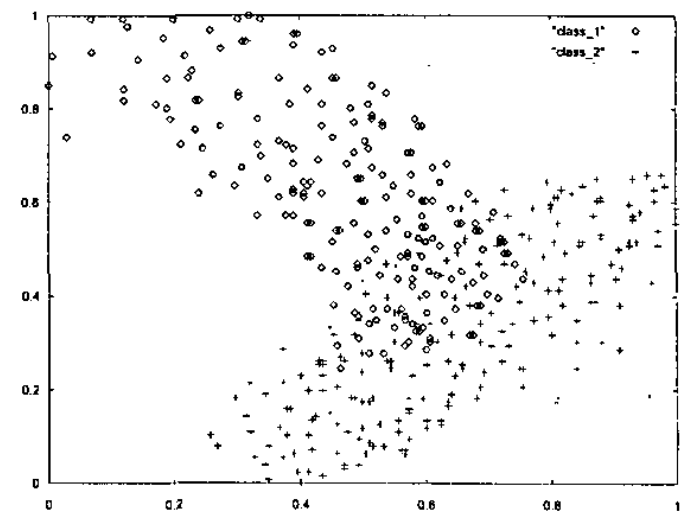

Fig. 9. Scatter plot of "T-shape" data

For this example, we choose initial $K$ values $\{9,11,13,15$, $17\}$ and assign initial memberships for the pattern set. Also, we choose $K=\{3,5,7,9,11\}$ for each $K-\mathrm{NN}$ method.

Fig. 10 show the average number of misclassifications for "T-shape" data for the fuzzy and interval type- 2 fuzzy $K-\mathrm{NN}$. As shown in Fig. 10, the interval type-2 fuzzy $K-\mathrm{NN}$ outperformed the fuzzy $K-\mathrm{NN}$.

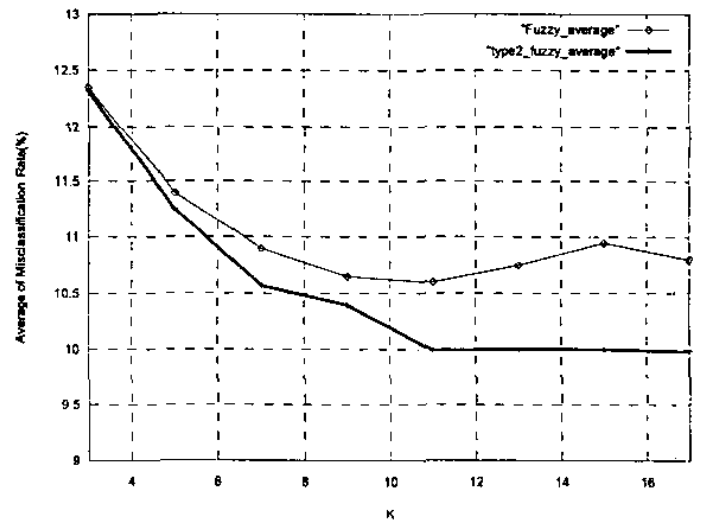

Fig. 10. Average misclassification rate for "T-shape" data

TABLE H

CONFUSION MATRIX FOR MINIMUM ERROR RATE

\begin{tabular}{ccccc}
\hline \multirow{2}{*}{ "T-shape" data } & \multicolumn{2}{c}{ Fuzzy } & \multicolumn{2}{c}{ Interval type-2 } \\
\cline { 2 - 5 } & Class 1 & Class 2 & Class 1 & Class 2 \\
\hline Class 1 & 219 & 9 & 220 & 8 \\
Class 2 & 32 & 187 & 30 & 189 \\
\hline
\end{tabular}

Table II shows the confusion matrix for the minimum error rate. As shown in the table, an improvement of a classification rate of $0.7 \%$ is achieved when we compare the 
best classification result for the fuzzy $K-\mathrm{NN}$ and interval fuzzy $K-\mathrm{NN}$.

'From the above graphs, we can conclude that the interval type-2 fuzzy $K-\mathrm{NN}$ is more desirable when we vary $K$. In other words, we may suggest that the classification result of interval type-2 fuzzy $K-\mathrm{NN}$ is more reliable than the fuzzy $K$ $\mathrm{NN}$ regardless of what value of initial $K$ we select.

\section{Conclusion}

In this paper, we presented an interval type-2 fuzzy $K$-NN algorithm that is an extension of the fuzzy $K-\mathrm{NN}$ algorithm that was proposed in [1]. In our proposed method, the membership values for each pattern were extended as interval type-2 fuzzy memberships by using several initial $K$. As a result, this method can handle and manage uncertainty that exist in choosing initial $K$. To show the effective of our method, we gave several results using the "twoclass" and "Tshape" data. As a further study, we plan to examine various data and also try to extend type-1 fuzzy memberships to the type-2 fuzzy memberships for various secondary grades.

\section{REFERENCES}

[1] J. Keller, M. Gray, and J. Givens, JR, "A fuzzy $K$ nearest neighbor algorithm," IEEE Trans. Syst., Man, Cybern., vol. 15, no. 4, pp. 258-263, August 1985

[2] J. Mendel, Uncertain Rule-Based Fuzzy Logic Systems: Introduction and New Directions. Prentice Hall, Upper Saddle River, NJ, 2001.

[3] J. Tou and R. Gonzalez, Pattern Recognition Principles. Addison-Wesley, 1974.

[4] J. Mendel and R. Bob John, "Type-2 fuzzy set made simple," IEEE trans. Fuzzy Syst., vol 10, no. 2, April 2002.

[5] J. Mendel and R. John, "A fundamental decomposition of type-2 fuzzy sets," 2001 IFSA/NAFIPS Joint Conference, pp. 1896-1901, Vancouver, BC, July 2001.

[6] N. Karnik and J. Mendel, "Type-2 fuzzy logic systems," IEEE Trans. Fuzzy Syst. vol. 7, no. 6, pp. 643-658, December 1999. 